\title{
Microscopic Findings Result Standard Unit
}

National Cancer Institute

\section{Source}

National Cancer Institute. Microscopic Findings Result Standard Unit. NCI Thesaurus.

Code C117586.

The standard unit of measure for microscopic findings results. 